\title{
Psoas Muscle Abscess
}

\author{
DANIEL ION ${ }^{1,2}$, BOGDAN SOCEA ${ }^{1,3}$, ALEXANDRA BOLOCAN ${ }^{1,2}$, DAN NICOLAE PĂDURARU ${ }^{1,2 *}$, \\ OCTAVIAN ANDRONIC ${ }^{1,2}$ \\ ${ }^{1}$ Carol Davila University of Medicine and Pharmacy, 37 Dionisie Lupu, 020021, Bucharest, Romania \\ ${ }^{2}$ Emergency University Hospital of Bucharest, 169 Splaiul Independentei, 050098, Bucharest, Romania \\ ${ }^{3}$ Sf. Pantelimon Emergency Clinical Hospital, 340 Sos. Pantelimon, 021659, Bucharest, Romania
}

Psoas muscle abcesses are a pathological entity, with very low incidence, and a lot of diagnosis and management discussions. Our paper aims to assess the presence of this pathology in literature as a short introductive narrative review and to present a series of cases from our experience. The research was retrospective, descriptive and enrolled a total of 14 patients. Specialty literature is poor regarding this pathology, with no agreement on the correct diagnosis and treatment algorithm. Future studies may offer diagnostic scores to facilitate rapid diagnosis.

Keywords: psoas muscle, abscess

Psoas muscle abcesses are a pathological entity, with very low incidence, and a lot of diagnosis and management discussions. Our paper aims to assess the presence of this pathology in literature as a short introductive narrative review and to present a number of cases from our experience.

\section{Anatomy}

Psoas muscle and iliac muscle are considered in specialized literature as a single muscle called iliopsoas, located in an extraperitoneal space called the iliopsoas compartment. The psoas muscle is long and fusiform, located laterally of the lumbar spine, on both sides. It passes under the inguinal ligament and anterior to coxo-femoral joint. It ends with tendinous insertion on the small trochanter of the proximal femural extremity. This muscle is innervated by the branches of the spinal nerves L2- L4 and is the most important flexor of the thigh [1]. Due to its localization, psoas muscle abscess is a common complication of vertebral tuberculosis [2,3].

Because of its position, the psoas muscle is adjacent to many organs: sigmoid colon, appendix, jejunum, ureter, abdominal aorta, kidney, pancreas, spine, and lymph nodes. Thus, an infectious process from any of these organs could diffuse into the psoas muscle, or infection at this level may occur by haematogenous spread from a anatomically far origin. These abscesses are located retrofascially, more than retroperitoneal, since the psoas muscle is posterior to the transversallis fascia, which represent the posterior border of the retroperitoneum. The incidence of this pathology is low, but has increased in recent years, with the widespread use of computer tomography in the diagnosis of various diseases.

\section{Clinical manifestations}

Two types of psoas muscle abscesses were described: primary and secondary. Primary abscesses appear due to lymphatic or haematogenous dissemination of a microorganism from a remote localized infection. The secondary occur because of the direct spread of an infectious neighborhood process [4].

Primary psoas muscle abscesses occur more frequently in children where they are often confused with hip joint septic arthritis, representing about $30 \%$ of all cases, or patients with other associated pathologies such as diabetes, chronic alcohol or intravenous drug users, kidney failure, history of malignancy or malnutrition [1]. Specialized literature describes the spread of infection from a remote infection (haematogenous) or a hematoma formed in the muscle that has become infected [5].

Secondary psoas muscle abscesses occur due to the near-close propagation of a localized infection from a close organ [1]:

- local infectious lymphadenitis

- infectious sacroileitis, osteomyelitis, septic arthritis

- infected femoral and abdominal aneurysm, catheterization of the important local vessels

- urinary tract infections, infected cancerous/noncancerous tumors, extracorporeal shock lithotripsy, intrauterine devices

*email:dan.paduraru,nicolae@gmail.com

All authors have contributed equally in writing, reading and approving the final manuscript. 
- colorectal pathology: cancer, Crohn's disease, diverticulitis, appendicitis

A complete and correct clinical examination is required to promptly diagnose this condition. If the patient maintains an antalgic position (patient is lying on his back, with slightly flexed thigh and rotated outward), the diagnostic is almost obvious $[1,4]$. This position lowers the pressure in the iliopsoas compartment and lowers the pain level.

The clinical picture presented by these patients is often variable and non-specific. The classic diagnostic triad: fever, lumbar pain and limp [6] is present in approximately $30 \%$ of patients [4]. Other symptoms may be diffuse abdominal pain, nausea and weight loss. Because of its location and innervation, the pain can irradiate to hips and thighs. The clinical picture may also include a pseudotumoral formation immediately below the inguinal ligament, which represents an element of differential diagnosis with femoral hernia.

There are also clear signs that mark the damage to the psoas muscle: the sign of the psoas. It is described as an increase in the intensity of abdominal pain at the passive flexion of the thigh with the patient in the dorsal decubitus. This sign has a sensitivity of $24 \%$ in the diagnosis of psoas abscesses [7].

\section{Microbiology}

Staphylococcus aureus is the most commonly identified microorganism in psoas abscesses, causing approximately $88 \%$ of primary abscesses [8]. Secondary psoas muscle abscesses are most often determined by germs belonging to Streptococcus $(4.9 \%)$ or E. coli $(2.8 \%)$. Mycobacterium tuberculosis is the most commonly reported organism in psoas abscesses that occur in patients in developing countries $[8,9]$. We can say that the pathogen that caused the infection of the primary organ is the same as the one that causes the abscess of psoas muscles in the case of secondary abscesses.

\section{Laboratory and imaging}

Before any treatment is administered, the patient may also present high white blood cells count, anemia, and elevated C-reactive protein levels accompanied by elevated ESR levels [10]. Blood cultures can also be positive for the same germ that has been identified in the abscess.

Initial radiological investigations may be targeted to diagnose other pathologies: intravenous urography, barium enema. These are, unfortunately, not specific to psoas abscesses and do not help in the diagnosis process. Sometimes, in the infection with anaerobic microorganisms, gas bubbles may appear in the retroperitoneum, which can establish the diagnostic [1].

A first-hand investigation may be the ultrasonography. It is easy to achieve and is not invasive for the patient. The disadvantage of this method is that it only visualizes psoas abscesses in approximately $60 \%$ of cases [9]. Retroperitoneal space may be difficult to visualize due to bowel gas [9]. The gold standard in the imaging diagnosis of psoas muscle abscesses is computerized tomography [1]. There are authors who claim that nuclear magnetic resonance would have far better results in the diagnosis of this pathology, because they can visualize the wall of the abscess without the need for intravenous contrast [1].

Although there are currently a multitude of imaging diagnostic methods for psoas muscle abscesses, there is still the possibility of a false negative diagnostic, so this pathology can be ignored by physicians [11].

\section{Treatment}

Treatment of psoas muscle abscesses is still a medical and surgical problem. According to other authors, there are several methods of treatment for psoas muscle abscesses: broad spectrum antibiotics, abscess drainage by CT or ultrasound guided puncture, surgical intervention and placement of a drainage tube at the cavity of the abscess [12, 13].

Often these methods intertwine. Because we have to administer targeted antibiotics, it is mandatory to obtain an abscess culture and also to know their sensitivity to antibiotics, which is why computed tomography guided percutaneous drainage or surgery is indicated to obtain biological material. In primary abscesses, most of the time there is also bacteremia, so blood cultures will be positive for the same microorganism.

At this moment, there are two types of treatment, which most often are used together: the appropriate course of antibiotics and drainage of the abscess. The initial choice of antibiotics is most often "blind". Usually broadspectrum antibiotics are administered as the first line of treatment until we have confirmation of the type and sensibility to antibiotics of the microorganism found in blood cultures or abscess fluid cultures. After we have confirmation of the microorganism causing the abscess, the treatment is shifted to the antibiotics that are most effective on the specific type of microorganism [9].

The treatment is different for primary versus secondary iliopsoas muscle abscesses. In primary abscesses antistaphylococal antibiotics should be administered before culture results [9]. In secondary abscesses, broadspectrum antibiotics should be administered before culture results (eg. antistaphylococal penicillin, aminoglycosides, clindamycin) [12]. 
There are 2 methods of drainage for iliopsoas muscle abscesses [1]:

1. Computed tomography guided percutaneous drainage - it is a less invasive procedure for the patient, with a low morbidity and mortality rate and it is classified in literature as the method of choice to drain this abscess [2, 14].

Associated with antibiotics, it can also be used as the initial treatment of iliopsoas muscle abscesses before surgery [1]. 2. Surgical drainage is indicated in a few well specified situations:

-When the computed tomography guided percutaneous drainage was unsuccessful.

-If the patient presents contraindications for the percutaneous drainage (clotting disorders, chronic anticoagulant treatment).

-If the patient has a different intraabdominal pathology that requires surgery (iliopsoas abscess due to Crohn's disease the affected bowel can also be removed in the same intervention) [15].

\section{Experimental part}

\section{Material and method}

This study analyzed a series of cases from an emergency surgical service at the Emergency University Hospital of Bucharest from January 2012 to January 2019, of the patients diagnosed with psoas abscess. The research was retrospective, descriptive and enrolled a total of 14 patients.

\section{Results}

From the demographic perspective, there were 7 men and 7 women, aged between 35-69 years. At the time of diagnosis, symptomatology was unspecific and covered fever (5 cases), abdominal pain ( 7 cases), lumbar pain (5 cases) and psoas sign (4 cases) (Table 1).

Table 1

DEMOGRAPHIC AND CLINICAL FEATURES OF THE STUDY GROUP

\begin{tabular}{|cccccccc|}
\hline Case no. & Year & Age & Sex & Fever & Abdominal pain & Lombar pain & Psoas sign \\
1 & 2012 & 35 & $\mathrm{M}$ & + & + & + & + \\
2 & 2012 & 69 & $\mathrm{M}$ & & + & + & + \\
3 & 2012 & 51 & $\mathrm{~F}$ & & + & + \\
4 & 2013 & 56 & $\mathrm{~F}$ & & + & + \\
5 & 2013 & 64 & $\mathrm{~F}$ & + & + & + \\
6 & 2013 & 63 & $\mathrm{M}$ & & + \\
7 & 2013 & 56 & $\mathrm{~F}$ & + & + & + \\
8 & 2014 & 52 & $\mathrm{M}$ & & + \\
9 & 2014 & 42 & $\mathrm{~F}$ & + & + & + \\
10 & 2014 & 61 & $\mathrm{M}$ & & + \\
11 & 2016 & 59 & $\mathrm{~F}$ & & + \\
12 & 2018 & 56 & $\mathrm{M}$ & & + \\
13 & 2018 & 59 & $\mathrm{~F}$ & + & + \\
+
\end{tabular}

Regarding the association of other pathologies in the patients from the study group, we identified the presence of type II diabetes mellitus in 7 patients and hypertension in 5 patients, obesity in 3 patients. The presence of renal disease (kidney failure/chronic kidney disease/renal lithiasis/urinary infection), the possible starting place for pathogens, has been identified in 6 patients. Spinal pathology was identified in a total of 5 patients with different levels (Table 2). 
Table 2

ASSOCIATED PATHOLOGIES OF THE PATIENTS IN THE STUDY GROUP

\begin{tabular}{|c|c|c|c|c|c|}
\hline Case no. & $\begin{array}{c}\text { Type II } \\
\text { diabetes } \\
\text { mellitus }\end{array}$ & Hypertension & Obesity & $\begin{array}{c}\text { Renal } \\
\text { pathology } \\
*\end{array}$ & $\begin{array}{c}\text { Spinal } \\
\text { pathology }\end{array}$ \\
\hline 1 & + & & & + & \\
\hline 2 & & + & + & & L2-L3 \\
\hline 3 & & + & & + & \\
\hline 4 & + & & & & \\
\hline 5 & & & & + & \\
\hline 6 & & + & & + & \\
\hline 7 & + & & + & & \\
\hline 8 & & & & & L4-L5 \\
\hline 9 & & & + & & \\
\hline 10 & + & & & & \\
\hline 11 & + & & & & L1-L2 \\
\hline 12 & + & + & & & \\
\hline 13 & + & & & + & L3-L4 \\
\hline 14 & & + & & + & L2-L3 \\
\hline
\end{tabular}

*kidney failure/chronic kidney disease/renal lithiasis/urinary infection

Laboratory results revealed leukocytosis in 10 patients, anemia in 6 patients, and thrombocytopenia in 1 patient. High fibrinogen level - a marker of inflammation - has been identified in a total of 13 patients. Serum creatinine was modified at 6 patients, so that urea had values above the maximum accepted in 8 patients. INR was significantly modified in 7 patients (Table 3 ).

The diagnosis of certainty was imagistic in all patients by computed tomography or IRM (Fig. 1).

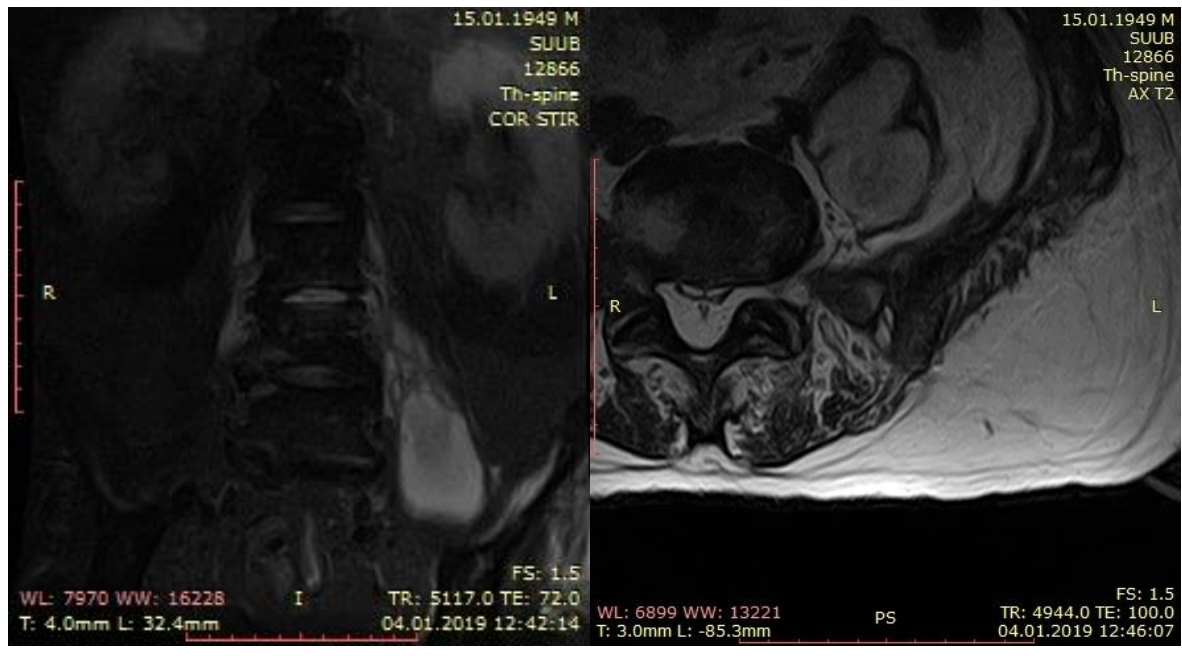

Fig. 1. IRM aspect of a psoas abcess of patient no. 14 
Table 3

PARACLINICAL AND BACTERIOLOGIC FEATURES OF THE STUDY GROUP

\begin{tabular}{|c|c|c|c|c|c|c|c|c|c|}
\hline Case no. & WBC & $\mathrm{Hb}$ & PLT & Fibrinogen & CREA & Uree & INR & $\begin{array}{c}\text { Surgical } \\
\text { approach* }\end{array}$ & Bacteriology \\
\hline 1 & 15.6 & 8.4 & 192 & 520 & 1.12 & 53 & 1.01 & median & - \\
\hline 2 & 9.8 & 12.4 & 165 & 420 & 0.69 & 20 & 2.10 & lombar & $\begin{array}{c}\text { Staphylococcus } \\
\text { aureus }\end{array}$ \\
\hline 3 & 19.5 & 11.6 & 210 & 887 & 1.02 & 51 & 1.13 & lombar & Acinobacter \\
\hline 4 & 9.4 & 14.2 & 116 & 776 & 1.61 & 51 & 1.59 & median & - \\
\hline 5 & 18.4 & 9.4 & 234 & 820 & 0.96 & 95 & 1.52 & lombar & $\begin{array}{l}\text { Staphylococcus } \\
\text { aureus + E.coli }\end{array}$ \\
\hline 6 & 8.6 & 9.1 & 184 & 301 & 3.80 & 28 & 1.24 & lombar & $\begin{array}{c}\text { Staphylococcus } \\
\text { aureus }\end{array}$ \\
\hline 7 & 17.5 & 11.9 & 196 & 543 & 0.65 & 20 & 1.04 & median & Acinobacter \\
\hline 8 & 22.3 & 13.1 & 212 & 899 & 0.99 & 36 & 1.74 & lombar & $\begin{array}{c}\text { Staphylococcus } \\
\text { aureus }\end{array}$ \\
\hline 9 & 8.4 & 9.5 & 164 & 861 & 1.09 & 42 & 1.20 & median & $\begin{array}{l}\text { Staphylococcus } \\
\text { aureus + E.coli }\end{array}$ \\
\hline 10 & 14.8 & 8.9 & 204 & 631 & 1.14 & 35 & 1.80 & lombar & - \\
\hline 11 & 17.25 & 11.2 & 173 & 889 & 0.59 & 30 & 1.22 & lombar & $\begin{array}{c}\text { Staphylococcus } \\
\text { aureus }\end{array}$ \\
\hline 12 & 16.1 & 12.1 & 162 & 708 & 0.9 & 80 & 1.24 & lombar & $\begin{array}{l}\text { streptococcus } \\
\text { pneumoniae }\end{array}$ \\
\hline 13 & 20.5 & 9.9 & 261 & 648 & 9.1 & 137 & 3.18 & lombar & $\begin{array}{c}\text { Staphylococcus } \\
\text { aureus }\end{array}$ \\
\hline 14 & 21.7 & 13.8 & 268 & 824 & 1.29 & 105 & 1.38 & lombar & $\begin{array}{c}\text { Staphylococcus } \\
\text { aureus }\end{array}$ \\
\hline
\end{tabular}

*median approach = laparotomy lombar approach = Leriche incision with extraperitoneal access

\section{Discussions and conclusions}

Psoas abscesses are a pathology with uncertainty adhesion to a particular specialty, which is why this entity is not mentioned as a distinct chapter of books. Often, it is an epiphenomenon or a complication of another pathology (renal, spinal, sigmoid) that confuses the specialist called initially to manage the case. Having clinical and paraclinical image with a difficult differential diagnosis in the context in which most patients have associated diseases that can lead to a presumptive diagnosis in another direction.

Differential diagnosis should be made with retroperitoneal tumors [16, 17], femoral hernia, enlarged inguinal nodes and iliac artery aneurysm [18].

In our research, most of the patients were initially hospitalized in other departments (nephrology, neurosurgery, internal medicine) and were transferred to our medical management service a few days after admission. This may delay the therapeutic gesture with adverse consequences on patient evolution. A multidisciplinary approach is mandatory in order to put the correct diagnosis in the shortest possible time.

The management should be optimized by the use of CT or IRM imaging investigations, which, in most cases, is decisive in establishing the therapeutic management. The guided drainage of psoas collections, although frequently cited in the literature, is rarely possible due either to low accessibility of the procedure, or to the imperative of the emergency of these cases.

The evolution of our patients was not influenced by the bacterial type identified in the pathogenic liquid from the psoas collection. The empiric antibiotherapy regimen was based on the Surgical Infection Society Guidelines, and included antimicrobial regimens for higher risk patients with intraabdominal infections [19]. We used the single agent regimen imipenem / cilastatin or meropenem, or combination regimens - aminoglycoside with metronidazole. The antibiogram obtained 72 hours postoperatively did not change the initial scheme.

Specialty literature is poor regarding this pathology, with low incidence, with no agreement on the correct diagnosis and treatment algorithm. Future studies may offer diagnostic scores to facilitate rapid diagnosis.

\section{References}

1. MALLICK IH, THOUFEEQ MH, RAJENDRAN TP, Postgrad. Med. J., 80, 2004, p. 459.

2. DINC H, ONDER C, TURHAN AU, ET AL, Eur. J. Radiol., 23, 1996, p. 130. 
3. CHERN CH, HU SC, KAO WF, ET AL, Am. J. Emerg. Med., 15, 1997, p. 83.

4. SHIELDS D, ROBINSON P, CROWLEY TP, Int. J. Surg., 10, no. 9, 2012, p. 466.

5. THONGNGARM T, MCMURRAY RW, Ann. Rheum. Dis., 60, 2001, p. 173.

6. LI Y, FUNAKOSHI H, SHIGA T, FUJITANI S, Cleveland Clinic Journal Of Medicine, 84, no. 11, 2017.

7. DEMUREN OA, ABOMELHA M, Saudi Med. J., 23, 2002, p. 96.

8. ARAI Y, KAWAKAMI T, SOGA H, ET AL, Int. J. Urol., 6, 1999, p. 257.

9. VAN DEN BERGE M, DE MARIE S, KUIPERS T, JANSZ AR, BRAVENBOER B, Neth. J. Med., 63, no. 10, 2005 , p. 413e6.

10. KUMAR S, Reviews in Medical Microbiology, 28, no. 1, 2017, p. 30.

11. RICCI MA, ROSE FB, MEYER KK, World J. Surg., 10, 1986, p. 834.

12. WONG OF, HO PL, LAM SK, Hong Kong Med. J., 19, no. 5, 2013, p. 416.

13. YIN HP, TSAI YA, LIAO SF, LIN PH, CHUANG TY, J. Chin. Med. Assoc., 67, 2004, p. 156.

14. TABRIZIAN P, NGUYEN SQ, GREENSTEIN A, RAJHBEHARRYSINGH U, DIVINO CM, Arch. Surg., 144, no. 10, 2009 , p. 946. doi:10.1001/archsurg.2009.144.

15. CANTASDEMIR M, KARA B, CEBI D, SELCUK ND, NUMAN F, Clin. Radiol., 58, no. 10, 2003, p. 811.

16. BRATU OG, MARCU RD, SOCEA B, NEAGU TP, DIACONU CC, SCARNECIU I, TURCU FL, RADAVOI GD, BRATILA E, BERCEANU C, SPINU AD, Rev. Chim. (Bucharest), 69, no. 7, 2018, p. 1813.

17. MARCU DR, IONITA-RADU F, IORGA LD, MANEA M, SOCEA B, SCARNECIU I, ISVORANU G, COSTACHE R, DIACONU CC, BRATU OG, Rev. Chim. (Bucharest), 70, no. 2, 2019, p. 445.

18. MARCU RD, DIACONU CC, CONSTANTIN T, SOCEA B, IONITA-RADU F, MISCHIANU DLD, BRATU OG, Experimental and Theraputic Medicine, doi: 10.3892/etm.2019, p.8020.

19. MAZUSKI JE, SAWYER RG, NATHENS AB, DIPIRO JT, SCHEIN M, KUDSK KA, YOWLER C, Surg. Infect. (Larchmt), 3, no. 3, 2002, p. 161.

$\overline{\text { Manuscript received: } 28.10 .2019}$ 